Case Report

\title{
Effect of Physical Exercise Training on Bullying and Depression in a Classroom: A Case Report
}

Arnaud Philippot ${ }^{1,2}$, Yannick Bleyenheuft ${ }^{1}$, Anne De Volder ${ }^{1,3, *}$

1. Institute of Neuroscience, MSL-In laboratory, Université catholique de Louvain, B-1200 Brussels, Belgium; E-Mails: arnaud.philippot@uclouvain.be; yannick.bleyenheuft@uclouvain.be; anne.de.volder@uclouvain.be

2. Psychiatric Hospital, AREA+, Epsylon ASBL, Brussels, Belgium

3. Pediatric Neurology Service, Cliniques Universitaires Saint-Luc, Brussels, Belgium

* Correspondence: Anne De Volder; E-Mail: anne.de.volder@uclouvain.be

Academic Editor: Gerhard Litscher

Special Issue: Integrative Therapies to Address Mood and Anxiety Symptoms in Youth and Young People

OBM Integrative and Complementary Medicine

2021, volume 6 , issue 1

doi:10.21926/obm.icm. 2101002
Received: June 21, 2020

Accepted: December 24, 2020

Published: January 07, 2021

\begin{abstract}
In a previous study conducted to assess the impact of physical exercise on the symptoms of depression and anxiety in preadolescents in the school environment, which included a subgroup of participants comprising eleven randomly selected teens (aged 9-11) and all enrolled in primary education, it was immediately observed that one participant referred to as Andy was being bullied and teased, and the teachers were completely unaware of this scenario. All the participants of the study were volunteers, and were subjected to low-tomoderate intensity exercise four times a week for five weeks. Psychological self-assessments and physical examinations were performed before and after the program as a part of a blind assessment. In the intervention sessions, the therapists and participants remained unaware of the individual scores. The sessions included the games that highlighted the feeling of movement, postural control, and slow relay races with balancing, in combination with other cooperative, interactive games focused on fun and social contact rather than focusing on the
\end{abstract}

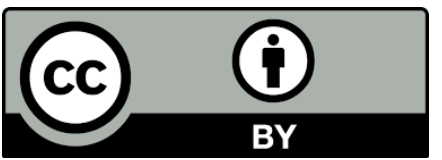

(C) 2021 by the author. This is an open access article distributed under the conditions of the Creative Commons by Attribution License, which permits unrestricted use, distribution, and reproduction in any medium or format, provided the original work is correctly cited. 
performance. It was reiterated to the participants that mockery was completely prohibited during the program. After the intervention, a significant decrease was observed in the anxiety levels and depression scores of the participants. The most significant change was observed in the participant who was a victim of bullying during the initial training sessions. No changes were observed in the general atmosphere of the classroom. Bullying is quite common ( $>10 \%)$ and is most often completely overlooked. The present study demonstrated a decrease in the symptoms of depression and anxiety in a non-clinical sample of pre-teens attending elementary school when they completed a low-to-moderate exercise program that combined movement and pleasure and encouraged positive and non-competitive interactions among the participants. The program proved to be particularly beneficial for a bullied individual.

\section{Keywords}

Exercise medicine; bullying; depression; preadolescents; school; physical training

\section{Introduction}

Bullying is a phenomenon that most students are exposed to during their school years. A metaanalysis estimated a mean prevalence of $35 \%$ for traditional bullying (including both victims and bullies) and $15 \%$ for cyberbullying among adolescents [1]. These children could be witnesses, victims, or the perpetrators of bullying. There is evidence that bullying critically affects the mental health development in the young student population. Bullies and the victims of bullying are at the risk of short-term and long-term negative impacts, such as depression, anxiety, low self-esteem, and delinquency [2-4]. It is noteworthy that young people already suffering from depressive symptoms might be more vulnerable to bullying because of the differences and the difficulties they experience in emotional regulation and coping [5]. Depression causes the affected child to be vulnerable and different from the peer group, thereby increasing their risk of being bullied. The condition of such children becomes worse as bullying by their peers adds to their discomfort. The symptoms of depression may worsen to a further severe level, leading to an increased risk of suicide. It is reported that bullying victimization may be a significant risk factor for adolescent suicide attempts worldwide [4], while depression may strongly influence this relationship between bullying and the risk of suicide [6]. The high prevalence of bullying and depressive symptoms among children and adolescents renders it imperative to manage this public health problem $[1,6]$. Furthermore, this situation is often associated with reduced physical activity leading to a severe sedentary lifestyle $[7,8]$ with worse long-term consequences developing in adulthood [9].

The phenomenon of bullying is quite common in the dynamics among peers within a classroom. In this context, group physical activity could serve as a support to children, assisting them to interact and learn new skills together, and improve their social skills and their ability to control their emotions. To date, few studies have examined the role of exercise interventions in reducing bullying incidences in school, with not a single study investigating depressive symptoms in addition to bullying. The limited number of studies available in the literature indicate that physical activity may mitigate the effects of bullying [10] and reduce depression [7]. In the underprivileged 
neighborhood of Santiago (Chile), a reduction in the level of bullying victimization was reported after participation in an 8-week-long physical activity intervention, compared to the control group. As stated in that report, the objective of the physical activity program was not limited to improving the physical condition of the participants, and included inculcating respect for rules, conflict resolution, positive interaction, and skill learning, all of which could explain, at least in part, the observed effect on bullying [11]. It is also reported that a reliable environment is essential to properly involve children and adolescents in physical activities [12]. These findings indicate that an efficient physical activity program in schools could prevent or reduce bullying within the classrooms, provided that the program is well-structured, task-oriented, safe, and noncompetitive.

We previously conducted a study to document the effectiveness of different training intensities in reducing the symptoms of depression and anxiety in pre-teens within school settings [13] and observed that low-to-moderate intensity physical exercise implemented in the form of games could reduce the scores for anxiety and depression in a non-clinical sample. The training program combined movement with pleasure and encouraged positive and non-competitive interactions among the participants. It was hypothesized that such a structured group physical activity program could influence the mood of the participants and alter the peer dynamics, and was, therefore, beneficial for all the participants, particularly the ones who are inhibited, insecure, and physically weak or non-athletic. One participant was bullied during the initial training sessions, which was inconsistent with the focus of the program to include movement and fun in a positive and non-competitive atmosphere. We, therefore, describe the case of this child, a boy (referred to as Andy) who became the victim of bullying, and his evolution through the course of this intervention. The objective of the present case report is to highlight the impact of this exercise program, over time, on Andy's well-being and fitness in parallel with the evolution of his peer group.

\section{Materials and Methods}

\subsection{General Design}

The details of the general design are available in a previous report [13]. Briefly, in a prospective and randomized pilot trial with two arms, two subgroups of participants, the randomly selected pre-teens (aged 9-11) attending school, thirteen in each group, were compared. The main objective of the trial was to study the effects of structured physical activity on the prevention or treatment of anxiety and depressive symptoms in a non-clinical sample of school children. Low-tomoderate-intensity physical activity was compared with high-intensity physical activity, to determine the most appropriate exercise intensity for application in depressed youth. In addition, the trial assessed whether such physical activities, implemented in the form of games, could affect peer dynamics and encourage the physically-weak children to participate in sport. There was no control group.

The low-to-moderate intensity intervention and the high-intensity intervention, both focused on pleasure, interaction, and non-competition, were conducted in parallel for five weeks at a rate of 4 sessions per week (Monday, Tuesday, Thursday, and Friday) during the lunch hour. Therefore, the study followed the two groups of children for 20 sessions that each lasted a total of $50 \mathrm{~min}$. 
There was an assessment period in the week before and after the program, in which test results of the participants were collected. The quantifiers for randomization included individual scores for the Child Depression Inventory (CDI), Body Mass Index (BMI), duration of the Léger shuttle test, age, sex, and school year. Thereafter, the principal investigator assorted the participants into two equivalent groups. Randomization at the group level was performed by an external blinded researcher, who provided the intervention allowance through e-mail. In the intervention sessions, the therapists and participants remained unaware of the individual scores used for randomization. The two pairs of therapists (two physical educators and two physiotherapists) managed an equal number of sessions in each group alternately.

The study was conducted at the "elementary school Parmentier-Jean XXIII" in Brussels and its facilities (a classroom and a gymnasium within the school premises, and a park surrounding the school). The project was conducted, including recruitments, evaluations, and experimental intervention periods, from November 2016 to December 2016, and was preceded by information sessions and a cooling-off period the month before. The study was approved by the Ethical Committee of the School of Medicine of the Université Catholique de Louvain ( $\mathrm{N}^{\circ}$. CEHF-FORM003/REV005-B403201627586), april 2016, revised and updated May, 20, 2020. The study is considered as a part of the clinical trial "Move and feel good" registred as NCT02970825, ClinicalTrials.gov, Autumn 2016 (https://clinicaltrials.gov/ct2/show/NCT02970825).

\subsection{Selection Process}

Participants were recruited from the primary school on a voluntary basis. The study was described inside classrooms, in the presence of teachers, and was presented to the parents in a separate session conducted at the school. The inclusion criteria for participation were as follows: (1) enrolled in an official education program; (2) aged between 9 and 12 years; (3) acceptance of the randomization principle; (4) absence of neurological or psychiatric history; (5) absence of uncorrected sensory disorder that could prevent the understanding of instructions; and (6) absence of conduct disorders. The exclusion criteria for the study were: (1) refusal to participate; (2) Body Mass Index above P95; (3) unstable diabetes; (4) coagulation disorders (besides specific exceptions and medical authorization); (5) severe and unstable asthma; (6) history of heart malformation or cardiovascular disease; and (7) medical conditions prohibiting sport or physical activity. No particular fitness level was a prerequisite for participation, and the teachers did not train the participants before or during the project. The parents of participants completed a medical questionnaire prior to the commencement of the research and also provided written informed consent for participation. Only the children who fulfilled the inclusion were allowed participation. All the participants were informed that they would not be allowed to select the group to which they would be assigned and that they could leave the study at any time if they wished.

\subsection{Intervention}

One of the two interventions was a low-to-moderate intensity physical exercise program. A greater reduction in the anxiety and depression scores was observed in this group compared to the other one, in which the participants followed high-intensity physical exercise [13]. 
The low-to-moderate intensity intervention focused on pleasure, positive interaction among the peers, and promotion of a general atmosphere that encouraged movement. A task-oriented climate with a learning component was promoted as it is reported to be associated with adaptive behaviors, healthy habits, and autonomous motivation to practice activities [14]. The intervention combined games that highlighted the feeling of movement, postural control, awareness of body, and slow relay races with balancing, mime games, and walk/run plays while perpetuating equilibration, and the other cooperative, interactive games focused on fun and social contact without a focus on performance. Each session began with a warm-up and ended with muscle stretching along with information and details regarding muscles. It was insisted repeatedly that mockery was completely prohibited during the program. The intervention was adapted to each participant to find an equivalent place for all the students. Positive remarks were regularly written in an individual's file to motivate each participant to attend all sessions ( 20 sessions in total). The principal investigator provided medical security and discussed the daily components of the program with the therapists, who were trained in physical education and physiotherapy from the Université catholique de Louvain, Belgium.

\subsection{Andy's Case (*)}

$\left({ }^{*}\right)$ This is a fictitious first name for use in the present report to maintain the anonymity of the student. It was observed during the initial training sessions that one participant was being bullied and teased, which the teachers were completely unaware of. This scenario was not known before the study commenced. In the initial sessions, it was observed that Andy had an inhibited behavior and stopped participating when the other participants referred to him as "Andy, handicap". When this was discussed with him separately, he informed that he encountered verbal mockeries in the classroom every day and that he felt excluded from everyone in the class. As a tall and thin figure, although not muscular, he was quite happy with his appearance. He disliked having trouble in making friends and did not seem to know why he failed in such attempts, except the guess that he was new to this school. He recognized that he was not sure of himself and his intelligence despite his good academic results. He was eleven years old, had no learning troubles, and his medical history was uneventful. He did not wish to inform his parents regarding his experience and was convinced that his teachers would do nothing to help him in this regard. We promised him that he would not have to experience bullying at least during the sessions, and "a bad atmosphere would imply the end of the intervention". We proposed referring to him as Drew (abbreviation for Andrew) during the sessions and everyone in the sessions followed. As the content of the intervention was adapted according to each child, it was also adapted to include Andy better without changing the principle of the physical exercise program. Each attempt to tease someone in the group was followed by a "You laugh, you need to relax" break (relaxation implied expulsion from the current play and one-minute individual neck massages by one of the physiotherapists). It was observed that during the subsequent sessions, Andy no longer had complaints and appeared happy to learn the new movements. The evolution of the mean scores obtained in the psychological and physical tests was analyzed for the group as well as individually for Andy. The aim was to determine the potential effect of such a program on bullying by analyzing the evolution of Andy's scores after five weeks of intervention. 


\subsection{Primary Outcomes}

Pre-assessments and post-assessments were conducted the week before and the week after the intervention, respectively, as a part of a blind assessment.

The Child Depression Inventory (CDI) is a 27-item self-reporting tool [15] with scores based on the child's statement describing how she or he felt during the two previous weeks. The average score in the general population is 9 points [15], and a score of 19 or above is considered pathological [16]. The CDI has good internal reliability (Cronbach's alpha $=0.86$ ), sensitivity, specificity, and a low level of bias [17].

The State-Trait Anxiety Inventory (STAI) [18] consists of two subscales, each with twenty items: the STAI form Y-A that assesses the participants' recent state (i.e., what the participants felt during the week of testing), and the STAI form Y-B that assesses the long-term anxiety trait (i.e., what the participants felt during the past year). Each part (A or B) of the STAI generates a state score or an anxiety trait score in the range of $20-80$ and the pathological threshold is $39-40$ [19]. STAI has a high degree of reliability (Cronbach's alpha $=0.86$ ) and validity.

\subsection{Secondary Outcomes}

A medical device and a scale were used to measure the height and weight, respectively, of the participants. The Leger shuttle test, a 20-meter multi-steps shuttle run test, was conducted to provide an extrapolated assessment of the maximal oxygen uptake $\left(\mathrm{VO}_{2}\right.$ max) [20]. A metaanalysis has reported that "the $20 \mathrm{~m}$ shuttle test seems to be a useful alternative to estimate cardio-respiratory fitness" and that the test has high validity in young people [21].

Harassment assessment was not conducted as the study was not formulated for that initially. However, a self-perception profile for children [22] was used in its French version to document the way each child assessed his skills (social, athletic, school, physical, behavioral, and self-esteem) before and after the intervention. After each session, the participants documented a quality assessment according to the two dimensions of well-being in an individual file: a "tiredness score" (0-3, 0: not tired; 1: a little tired; 2: moderately tired; 3: exhausted) and a "pleasure index" (0-3, 0: very fun; 1 : quite fun; 2 : moderately boring; 3 : very boring). This file was referred to when adapting the subsequent physical exercise sessions according to each participant.

\subsection{Statistical Analysis}

As certain measures were based on Likert scores, a graphical tool (quantile-quantile plot) and a Kolmogorov Smirnov test were employed to assess the distribution of normality. If the data were not normal, a logarithmic transformation was performed to obtain the normalized data (if that was possible). As it was assumed that there would be changes in the depression/anxiety scores after the intervention, the dependent tests (Student's paired t-test or Wilcoxon's test) were performed to determine the change in a variable (STAI, CDI, etc.) over time. All the statistical procedures were performed using the SPSS software. 


\section{Results}

\subsection{Group Evolution}

Among the 13 preadolescents recruited in the study, two were excluded during the experiment; one withdrew voluntarily and the other was excluded on account of bad behavior (opposition to the exercise instructions). The statistical analysis of the pre-test and post-test measures was performed for the remaining 11 participants. There were five girls and six boys, and the mean age at assessment was $10.6 \pm 0.7$ years. The mean attendance rate was $96.65 \%$. The mean individual "tiredness score" was low (0.43). The participants enjoyed the intervention, as evidenced by the mean "fun index" value of 0.52 (very fun to quite fun).

The self-reported data from the primary questionnaires are summarized in Table 1. The Likert score variable that demonstrated a non-normal distribution was the CDI. However, the CDI scores were transformed using the formula "Ln(variable)" to obtain a normal distribution.

Table 1 Mean scores obtained in psychological self-assessments and physical tests.

\begin{tabular}{llllll}
\hline Variables & Time & $\mathrm{N}$ & Mean & SEM & $\begin{array}{l}\text { p- } \\
\text { value }^{\circ}\end{array}$ \\
\hline STAI A & T1 & 11 & 30.55 & 2.60 & .628 \\
STAI B & T2 & 11 & 29.36 & 2.04 & \\
& T1 & 11 & 38.82 & 2.20 & .004 \\
CDI & T2 & 11 & 33.36 & 2.83 & \\
& T1 & 11 & 10.36 & 2.83 & .006 \\
Height $(\mathrm{cm})$ & T2 & 11 & 6.73 & 1.88 & \\
& T1 & 11 & 139.72 & 3.05 & .001 \\
Weight $(\mathrm{kg})$ & T2 & 11 & 140.27 & 3.12 & \\
& T1 & 11 & 34.28 & 1,74 & .004 \\
BMI $\left(\mathrm{kg} / \mathrm{m}^{2}\right)$ & T2 & 11 & 35.00 & 1.74 & \\
& T1 & 11 & 17.49 & 0.54 & .149 \\
VO ${ }_{2}$ Max $(\mathrm{mL} / \mathrm{min} / \mathrm{kg})$ & T2 & 11 & 17.97 & 0.54 & \\
& T2 & 11 & 42.45 & 1.34 & .738 \\
Age & & 11 & 42.89 & 1.77 & \\
\hline
\end{tabular}

Legend for Table 1. P-value $<0.05$ is considered significant; SEM: standard error of the mean; ${ }^{\circ} \mathrm{p}$-value in dependent test. Modified from ([12] doi: 10.3389/fpsyg.2019.01820. The cited original work is licensed under the Creative Commons Attribution 4.0 International License.

The STAI B scores, on an average, decreased from 38.82 [SEM (Standard Error of Mean) 2.20] at T1 to 33.36 (SEM 2.83) at T2 ( $p=0.004$, Student's paired t-test) [refer to Table 1].

The analysis of the STAI-A scores did not reveal any time-dependent change ( $p$-value $>0.05$ ).

The CDI scores decreased, on average, from 10.36 (SEM 2.83) at T1 to 6.73 (SEM 1.88) at T2 ( $p$ $=0.006$, Student's paired t-test, refer to Table 1 and Figure 1). 


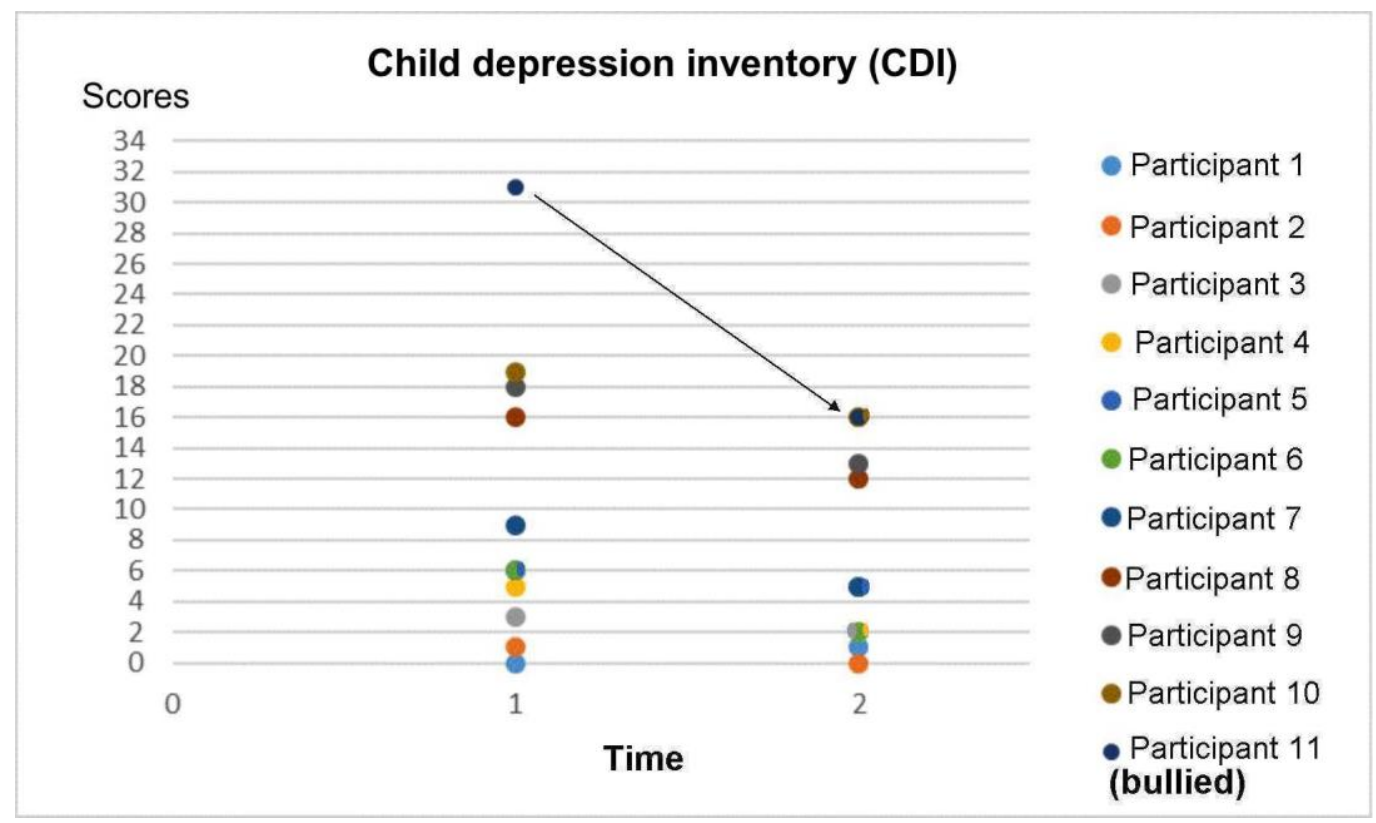

Figure 1 Individual scores in the Child Depression Inventory (CDI). At the group level, a significant $(p=0.006)$ reduction in the CDI scores was observed after five weeks of intervention. The individual score for Andy, the child who was bullied in the beginning, fell below the set threshold and was closer to the scores for other children after the intervention. The pathological threshold was set at 19.

A Student's paired t-test was performed on the VO2 max estimations that were evaluated using the Léger shuttle test, and no significant changes were revealed. The scores remained stable in general, evolving from 42.45 (SEM 1.34) $\mathrm{mL} / \mathrm{min} / \mathrm{kg}$ at T1 to 42.89 (SEM 1.77) $\mathrm{mL} / \mathrm{min} / \mathrm{kg}$ at T2. The weight and height measures demonstrated significant changes, while the BMI score remained stable (refer to Table 1).

\subsection{Andy's Evolution}

The statistical analysis was conducted anonymously at the group level. A posteriori, it was observed that Andy had the most severe scores in the group for the symptoms of depression, as assessed by the CDI test, with a pathological score of 31 before the intervention, which was way higher than the cut-off score of 19 set for the test. Andy also appeared to be suffering from a low level of anxiety, with scores of 38 and 43 for anxiety in state and trait, respectively. Prior to the intervention, Andy obtained the lowest result in the physical test, with an estimate of the $\mathrm{VO}_{2} \mathrm{Max}$ of $30.35 \mathrm{~mL} / \mathrm{min} / \mathrm{kg}$. According to the international standards of the $20 \mathrm{~m}$ shuttle test [23], Andy was in the lowest 5 percentiles as per the international norms for boys. Table 2 lists all the scores of Andy.

Table 2 Andy's scores in psychological self-assessments and physical tests.

\begin{tabular}{lll}
\hline Variables & Time & Mean \\
\hline STAI A & T1 & 38 \\
& T2 & 35 \\
\hline
\end{tabular}




\begin{tabular}{lll}
\hline STAI B & T1 & 43 \\
CDI & T2 & 43 \\
& T1 & 31 \\
Height $(\mathrm{cm})$ & T2 & 16 \\
Weight $(\mathrm{kg})$ & T1 & 127 \\
& T2 & 127 \\
BMI $\left(\mathrm{kg} / \mathrm{m}^{2}\right)$ & $\mathrm{T} 1$ & 25.7 \\
& T2 & 27.0 \\
VO ${ }_{2} \mathrm{Max}(\mathrm{mL} / \mathrm{min} / \mathrm{kg})$ & $\mathrm{T} 1$ & 15.9 \\
& $\mathrm{~T} 2$ & 16.7 \\
Age & $\mathrm{T} 1$ & 30.35 \\
\hline
\end{tabular}

After the intervention, Andy's depressive symptoms fell below the pathological threshold, with a lower score of 16 , which was closer to that of the other children in the group (Figure 1). Andy then remained relatively stable in terms of the evolution of anxiety symptoms.

Andy had benefited from the program on a physical basis as well, as evidenced by his improved cardio-respiratory score. His estimated $\mathrm{VO}_{2} \mathrm{Max}$ was improved by $1.47 \mathrm{~mL} / \mathrm{min} / \mathrm{kg}$, and he was no longer the weakest member of the group.

The changes in Andy's responses in the self-perception profile for children indicated improved self-esteem in the athletic and behavioral dimensions.

\section{Discussion}

The present study aimed to introduce an original intervention for physical exercise in schools and assess the potential effects of this intervention on the symptoms of depression and anxiety in the participating children, including the child who was bullied. It was observed that physical exercise at low-to-moderate intensity implemented in the form of games could reduce the anxiety and depression scores in this non-clinical sample and exerted a beneficial impact over time on the mental and physical health of the bullied participant in parallel with the evolution of the group.

\subsection{Group Evolution}

The present study demonstrated that a five-week-long program of physical exercise at a low-tomoderate intensity could reduce the symptoms of depression and anxiety in a non-clinical sample of pre-teens attending elementary primary school. In addition to the beneficial effects on the whole group, the intervention appeared to be particularly beneficial for a bullied individual. There are several potential explanations for this observation of improved mood in the peer group as a whole. First, the program provided a positive experience of movement and peer interaction with the inclusion of all children. The intervention included physical activities without a sense of competition and not requiring a demonstration of performance. The sessions were conducted in a group, allowing the children to collaborate and form teams with the common goal of executing the suggested movements accurately. Second, the program, conducted within the school premises, was appreciated as recreational and full of fun. A high rate of participation (96.65\%) despite the 
voluntary nature of participation highlighted the importance of engaging children. It is reported that children, mostly girls, increased their level of participation in physical activities when a motivational and task-oriented climate was established [24]. In our study also, we promoted a task-oriented climate with a learning component as such an environment is reported to be more associated with adaptive behaviors, healthy habits, and autonomous motivation to practice sports outside of school [24]. The interventions based on light-to-moderate physical activity, body awareness, and yoga practice may have synergizing benefits, reducing the negative effects of chronic stress [25]. On the contrary, physical exercise programs conducted in an ego-oriented climate could lead to an extrinsic motivation, which is associated with further abandonment of physical activity [24]. Third, physical activity might be effective in decreasing bullying tendencies in children. In a cohort study with 1,248 high-school students, a trend was observed that the teenagers who regularly engaged in physical activity could alter their levels of aggressivity more than those with sedentary behavior. This led the authors to suggest the promotion of healthy (non-competitive) sport to prevent violence among children [26]. Another hypothesis could be that physical exercise reduces depressive symptoms and improves mental well-being among children through its beneficial effects on brain functions. Previous authors have demonstrated that in the general young population, physical exercise improves the performance of executive functions and modifies the activation pattern of the brain areas underlying these functions [27, 28]. Cognitive and executive functions are often impaired in depressed young people [29, 30]. To date, there are no published studies on the impact of exercise on the brain in the children and adolescents with depressive symptoms. A few systematic reviews and meta-analyses have evidenced that exercise interventions reduce the symptoms of depression in children and adolescents, with a greater impact on the clinical symptoms [31, 32]. Nonetheless, additional studies with better methodological quality are required to corroborate and confirm these results, particularly in school settings.

\subsection{Andy's Evolution}

Prior to the intervention, Andy obtained a considerably high score on the depression scoring scale (CDI). This observation was consistent with the findings of a recent study on the association between bullying and depressive symptoms conducted with 4,829 adolescents [10]. Although Andy did not express morbid ideas, it has been long known that bullies, victims, and bully-victims are at risk of suffering from depressive symptoms that can even lead to suicide in certain cases [10]. In the early sessions, the bullying was clearly influenced by both his inhibited individual behavior and the dynamics of an already-formed group in which he was new and poorly accepted because of these personal characteristics. The intervention was adapted to include him better and foster a non-competitive and benevolent climate where he could participate at the same level as the other children of the group and acquire new skills with pleasure without focusing on his performance. After the intervention, it was observed that Andy was the one to make the most of this kind of physical exercise program. As evidenced by his individual score, Andy felt much better after the intervention, obtaining a score under the pathological threshold score. The intervention appeared to have exerted a beneficial impact on his mood. It is reported that participation in sports may be an effective way to reduce the impact of bullying on the risk of developing depression [10]. A previous study demonstrated that physical activity at moderate-to-vigorous 
intensity for eight weeks resulted in reduced physical and verbal victimization among school children (aged 8-10 years) [11]. Furthermore, a cohort study with a sample of 29,207 adolescents from private and public high schools demonstrated that exercise reduced sadness and suicidal ideation in adolescents who were bullied at school [33, 34]. Our findings are consistent with these previously reported observations, although it must be noted that we report no objective measure that could indicate that Andy was no longer bullied in school after the program. We had access to the reduction in his depressive symptoms only until immediately after the intervention. Further studies based in school settings are clearly required to elucidate the extent to which such a program could result in the prevention or treatment of bullying.

\subsection{Physical Activity: A Tool for use within a Reliable Environment}

It is noteworthy that physical activity may provide opportunities for bullying to occur. In turn, bullying may cause reduced participation in physical activity [35]. If students develop a negative feeling regarding sports activity, they would have a negative experience and would ultimately reject the desire and motivation to participate in any physical activity. Therefore, it is advised to maintain a motivational atmosphere that is adapted according to the child to prevent intimidation behaviors and support positive interaction [24]. On similar lines, a critical qualitative study conducted with a sample of 15 boys explored the social-behavior mechanisms that motivate or dissuade participation in physical activities at the dawn of adolescence [36]. Studies have identified different mediation mechanisms to promote participation in physical activity, such as emotions, the pleasure of moving, and the feeling of connection to the activity. While negative self-perceptions, unsatisfaction with own body, repeated negative experiences, and bullying are reported to lead to apathy and attrition from physical exercise [36], a safe atmosphere provided by the monitors, an atmosphere of motivation oriented toward the task [24], and sessions focused on enjoyment motivate the children and even those with a potential reluctance to participate [36]. The most appropriate intensity and frequency of exercise for reducing depressive symptoms are not yet clearly defined [37], although there is good evidence demonstrating that moderateintensity exercise reduces depression [31,32,38]. A mild-to-moderate or moderate level of intensity may be required when the objectives of the intervention are to promote positive interaction, collaboration among the participants, and improvement in their physical condition. Young people who are depressed might experience more difficulty in performing the usual tasks. Therefore, high-intensity tasks may appear like a huge burden and effort to them, lowering their available capacities to collaborate in the program and interact with the other participants. In overly competitive sports clubs, this might encourage intimidation from assertive participants toward their vulnerable peers. On the contrary, low-to-moderate intensity would be a convenient way to gain the experience of success, encouraging adherence to the exercise regimen. In our opinion, such a program would increase the likelihood of young people adhering to exercise for a longer period of time, thereby facilitating the prevention of all kinds of physical and mental illnesses. Nonetheless, additional studies with good methodological quality are necessary to corroborate and confirm these results, particularly in school settings. A few systematic reviews and meta-analyses have already evidenced that physical exercise interventions reduced the depressive symptoms in children and adolescents compared to control groups, with a higher impact on clinical symptoms [31, 32]. The limited number of meta-analyses available in the 
literature present a high level of inter-study heterogeneity in terms of the intervention methods and the measurement of results, particularly apparent in adolescents [31, 37]. The present study observed that if children, including the victims of bullying, followed an adapted physical exercise program, they could achieve a positive development in their well-being. We observed a reduction in the depression scores of children after five weeks of low-to-moderate intensity physical exercise program conducted in a non-competitive learning-climate that favored peer interaction. The current public fitness rooms are generally not adapted to realize this goal. Further studies in school settings are clearly required to elucidate the extent to which such a program could assist in the prevention or treatment of bullying.

\subsection{COVID-19 Context of School Closures}

The COVID-19 pandemic crisis has significantly affected numerous students worldwide. The lockdown restrictions force the students to undertake their school programs online and also limit their social life. Students often experience fears and uncertainties with the ongoing physical and social isolation, which puts them at potential risk of cyberbullying. Children and adolescents react differently as they are at different stages of development, although higher rates of depression, anxiety, and post-traumatic symptoms are being reported across all age groups [39, 40]. In regard to the preventive measures required to be adopted concerning the mental health of students, a rapid systematic review demonstrated the protective effect of a routine of physical activity during the COVID-19 period. Maintaining physical fitness has also been demonstrated to reduce the risk of developing symptoms of depression by $12 \%-32 \%$ and the risk of experiencing anxiety by $15 \%-$ $34 \%$ in the general population [41]. Therefore, during forced isolation, such as the one during the pandemic crisis, certain authors have suggested studying the role of online exercise programs, such as the \#StayHomeStayFit project by the University of Milan [42], in promoting fitness and preventing mental health disorders [41].

A few countries have decided to keep the schools open. In keeping with health precautions, it would be desirable to evaluate the maintenance of physical education classes through inexpensive, feasible, and effective programs. Racket sports, climbing, fitness, and aerobic games can be conducted in a ventilated room or outdoors with distance adjusted according to the intensity of the exercise and while wearing a mask. However, to date, it has proved difficult, in practice, to estimate the distance necessary to avoid the transmission of infection during a rigorous sport.

\subsection{Limitations}

As with all research, the present study also has certain limitations, particularly in the interpretation of results. First, we did not expect that a child would be bullied during this experiment. The bullying event was revealed during the initial sessions when Andy, after being teased and bullied, informed us regarding his situation. We present him in this study as a special case as the results obtained related to his evolution were encouraging and could assist in the investigation of the impact of a physical activity intervention on the well-being of a bullied child. However, these results should be interpreted with caution. No harassment assessment [43, 44] was performed in this trial as the trial was initially not designed to collect the measures regarding bullying of participants. Only the data collected from the primary study has been discussed [13]. The long-term effects of the treatment could be determined by analyzing the psychological scores 
and the class atmosphere after the intervention, although this was not realized as there was no follow-up and we had only one window of five weeks. The study included only 11 participants who completed the whole study and this small sample was not sufficient to explore whether the course was different depending on the social characteristics of the participants. Moreover, we did not include a control group without intervention. Further experimental interventions involving physical exercise should be conducted to address the issue of bullying and assess mental health in children, including tests that would describe the bullying/aggressor position for each child. The general atmosphere in the classroom and the family environment [2] should also be considered while measuring the potential impact of physical exercise in different situations.

\section{Conclusion}

The present study indicated that the symptoms of depression were reduced in a non-clinical sample of 11 school children, including the case of a victim of bullying, when the children were on a low-to-moderate intensity exercise program that combined movement and pleasure in a taskoriented and non-competitive climate. Further comparative and randomized studies are necessary to document the reduction in depression in the context of bullying after conducting an intervention involving physical activities adapted according to school to promote physical exercise as care.

\section{Acknowledgments}

We would like to thank Mr. Olivier Parmentier for his sponsorship and support to our project by graciously providing the summer equipment of Park Parmentier. We thank the director and staff of the school for the great welcome. We wish to thank Prof. Vincent Dubois for the financial support to A.P. by the sponsorship of the Baillet-Latour asbl Funds (Belgium). AG De Volder is Senior Research Associate at the Belgian National Fund for Scientific Research.

\section{Author Contributions}

AP contributed to the study design, was involved in the exercise intervention trial, made the statistical analysis of data, and wrote the first draft of the manuscript. YB contributed to the study design and manuscript draft. ADV made the study design, verified the exercise intervention, made all scoring, provided results on an anonymous basis, and wrote the manuscript. All authors had access to the study data that support the publication.

\section{Funding}

This study was supported by a Bosquet-Polak Prize (Belgium) to ADV.

\section{Competing Interests}

The authors have declared that no competing interests exist.

\section{Reference}

1. Modecki KL, Minchin J, Harbaugh AG, Guerra NG, Runions KC. Bullying prevalence across 
contexts: a meta-analysis measuring cyber and traditional bullying. J Adolesc Health. 2014; 55: 602-611.

2. Shetgiri R. Bullying and victimization among children. Adv Pediatr. 2013; 60: 33.

3. Holt MK, Vivolo-Kantor AM, Polanin JR, Holland KM, DeGue S, Matjasko JL, et al. Bullying and suicidal ideation and behaviors: A meta-analysis. Pediatrics. 2015; 135: e496-e509.

4. Koyanagi A, Oh H, Carvalho AF, Smith L, Haro JM, Vancampfort D, et al. Bullying victimization and suicide attempt among adolescents aged 12-15 years from 48 countries. J Am Acad Child Adolesc Psychiatry. 2019; 58: 907-918.e4.

5. Menesini E, Salmivalli C. Bullying in schools: The state of knowledge and effective interventions. Psychology Health Med. 2017; 22: 240-253.

6. Kodish T, Herres J, Shearer A, Atte T, Fein J, Diamond G. Bullying, depression, and suicide risk in a pediatric primary care sample. Crisis. 2016; 37: 241-246.

7. Korczak DJ, Madigan S, Colasanto M. Children's physical activity and depression: A metaanalysis. Pediatrics. 2017; 139: e20162266.

8. Mehler-Wex C, Kölch M. Depression in children and adolescents. Dtsch Arztebl Int. 2008; 105: 149-155.

9. Korczak DJ, Ofner M, LeBlanc J, Wong S, Feldman M, Parkin PC. Major Depressive Disorder Among Preadolescent Canadian Children: Rare Disorder or Rarely Detected? Acad Pediatr. 2017; 17: 191-197.

10. Holbrook HM, Voller F, Castellini G, Silvestri C, Ricca V, Cassioli E, et al. Sport participation moderates association between bullying and depressive symptoms in Italian adolescents. J Affect Disord. 2020; 271: 33-38.

11. Hormazábal-Aguayo I, Fernández-Vergara O, González-Calderón N, Vicencio-Rojas F, RussellGuzmán J, Chacana-Cañas C, et al. Can a before-school physical activity program decrease bullying victimization in disadvantaged children? The Active-Start Study. Int J Clin Health Psychol. 2019; 19: 237-242.

12. Mitchell TB, Cooley JL, Evans SC, Fite PJ. The moderating effect of physical activity on the association between ADHD symptoms and peer victimization in middle childhood. Child Psychiatry Hum Dev. 2016; 47: 871-882.

13. Philippot A, Meerschaut A, Danneaux L, Smal G, Bleyenheuft Y, De Volder AG. Impact of physical exercise on symptoms of depression and anxiety in pre-adolescents: A pilot randomized trial. Front Psychol. 2019; 10: 1820.

14. Cid L, Pires A, Borrego C, Duarte-Mendes P, Teixeira DS, Moutão JM, et al. Motivational determinants of physical education grades and the intention to practice sport in the future. PLoS One. 2019; 14: e0217218.

15. Kovacs M, Beck AT. Cognitive-affective processes in depression. Emotions in Personality and Psychopathology. Boston, MA: Springer US; 1979. p.415-p.442.

16. Smucker MR, Craighead WE, Craighead LW, Green BJ. Normative and reliability data for the Children's Depression Inventory. J Abnorm Child Psychol. 1986; 14: 25-39.

17. Stockings E, Degenhardt L, Lee YY, Mihalopoulos C, Liu A, Hobbs M, et al. Symptom screening scales for detecting major depressive disorder in children and adolescents: A systematic review and meta-analysis of reliability, validity and diagnostic utility. J Affect Disord. 2015; 174: 447-463.

18. Spielberg C, Gorsuch R, Lushene R, Vagg PR, Jacobs GA. Manual for the State-Trait Anxiety 
Inventory (STAI Form Y). Paris: Les Editions du Centre de Psychologie Appliquée; 1983.

19. Knight RG, Waal-Manning HJ, Spears GF. Some norms and reliability data for the State--Trait Anxiety Inventory and the Zung Self-Rating Depression scale. Br J Clin Psychol. 1983; 22: 245249.

20. Leger LA, Mercier D, Gadoury C, Lambert J. The multistage 20 metre shuttle run test for aerobic fitness. J Sports Sci. 1988; 6: 93-101.

21. Mayorga-Vega D, Aguilar-Soto P, Viciana J. Criterion-related validity of the 20-m shuttle run test for estimating cardiorespiratory fitness: A meta-analysis. J Sports Sci Med. 2015; 14: 536547.

22. Harter S. Manual for the Self-perception Profile for Children:(Revision of the Perceived Competence Scale for Children). Denver, CO: University of Denver; 1985.

23. Tomkinson GR, Lang JJ, Tremblay MS, Dale M, LeBlanc AG, Belanger K, et al. International normative $20 \mathrm{~m}$ shuttle run values from 1142026 children and youth representing 50 countries. Br J Sports Med. 2017; 51: 1545-1554.

24. Castro-Sánchez M, Chacón-Cuberos R, Ubago-Jiménez JL, Zafra-Santos E, Zurita-Ortega F. An explanatory model for the relationship between motivation in sport, victimization, and video game use in schoolchildren. Int J Environ Res Public Health. 2018; 15: 1866.

25. D'Alessio L, Korman GP, Sarudiansky M, Guelman LR, Scévola L, Pastore A, et al. Reducing allostatic load in depression and anxiety disorders: Physical activity and yoga practice as addon therapies. Front Psychiatry. 2020; 11: 501.

26. Méndez I, Ruiz-Esteban C, Ortega E. Impact of the physical activity on bullying. Front Psychol. 2019; 10: 1520.

27. Chaddock L, Erickson KI, Prakash RS, Kim JS, Voss MW, Vanpatter M, et al. A neuroimaging investigation of the association between aerobic fitness, hippocampal volume, and memory performance in preadolescent children. Brain Res. 2010; 1358: 172-183.

28. Voss MW, Chaddock L, Kim JS, Vanpatter M, Pontifex MB, Raine LB, et al. Aerobic fitness is associated with greater efficiency of the network underlying cognitive control in preadolescent children. Neuroscience. 2011; 199: 166-176.

29. Belzung C, Willner P, Philippot P. Depression: From psychopathology to pathophysiology. Curr Opin Neurobiol. 2015; 30: 24-30.

30. Wagner S, Muller C, Helmreich I, Huss M, Tadic A. A meta-analysis of cognitive functions in children and adolescents with major depressive disorder. Eur Child Adolesc Psychiatry. 2015; 24: 5-19.

31. Carter T, Morres ID, Meade O, Callaghan P. The effect of exercise on depressive symptoms in adolescents: A systematic review and meta-analysis. J Am Acad Child Adolesc Psychiatry. 2016; 55: 580-590.

32. Radovic S, Gordon MS, Melvin GA. Should we recommend exercise to adolescents with depressive symptoms? A meta-analysis. J Paediatr Child Health. 2017; 53: 214-220.

33. Sibold J, Edwards E, Murray-Close D, Hudziak JJ. Physical activity, sadness, and suicidality in bullied US adolescents. J Am Acad Child Adolesc Psychiatry. 2015; 54: 808-815.

34. Sibold J, Edwards EM, O'Neil L, Murray-Close D, Hudziak JJ. Bullying environment moderates the relationship between exercise and mental health in bullied US children. J Sch Health. 2020; 90: 194-199.

35. Pulido R, Banks C, Ragan K, Pang D, Blake JJ, Mckyer EL. The impact of school bullying on 
physical activity in overweight youth: Exploring race and ethnic differences. J Sch Health. 2019; 89: 319-327.

36. Jachyra P, Gibson BE. Boys, transitions, and physical (in) activity: Exploring the sociobehavioural mediators of participation. Physiother Can. 2016; 68: 81-89.

37. Larun L, Nordheim LV, Ekeland E, Hagen KB, Heian F. Exercise in prevention and treatment of anxiety and depression among children and young people. Cochrane Database Syst Rev. 2006: CD004691.

38. Oberste $M$, Medele $M$, Javelle $F$, Lioba Wunram $H$, Walter $D$, Bloch $W$, et al. Physical activity for the treatment of adolescent depression: A systematic review and meta-analysis. Front Physiol. 2020; 11: 185.

39. Marques de Miranda D, da Silva Athanasio B, Sena Oliveira AC, Simoes-e-Silva AC. How is COVID-19 pandemic impacting mental health of children and adolescents? Int J Disaster Risk Reduct. 2020; 51: 101845.

40. Jiao WY, Wang LN, Liu J, Fang SF, Jiao FY, Pettoello-Mantovani M, et al. Behavioral and emotional disorders in children during the COVID-19 epidemic. J Pediatr. 2020; 221: 264266.e1.

41. Wolf S, Zeibig J, Seiffer B, Welkerling J, Brokmeier L, Atrott B, et al. Can physical activity protect against depression and anxiety during the COVID-19 pandemic? A rapid systematic review. 2020. doi: 10.21203/rs.3.rs-81150/v1

42. Lucini D, Gandolfi CE, Antonucci C, Cavagna A, Valzano E, Botta E, et al. \#StayHomeStayFit: UNIMI's approach to online healthy lifestyle promotion during the COVID-19 pandemic. Acta Biomed. 2020; 91: e2020037.

43. Slater A, Tiggemann M. Gender differences in adolescent sport participation, teasing, selfobjectification and body image concerns. J Adolesc. 2011; 34: 455-463.

44. Dias DT, Claumann GS, Ribovski M, Folle A, Farias GO, Silva DAS, et al. Cross-Cultural adaptation to Brazilian portuguese of an instrument to assess teasing during physiCal/sports aCtivity among Brazilian adolesCents. Rev Paul Pediatr. 2018; 36: 329.

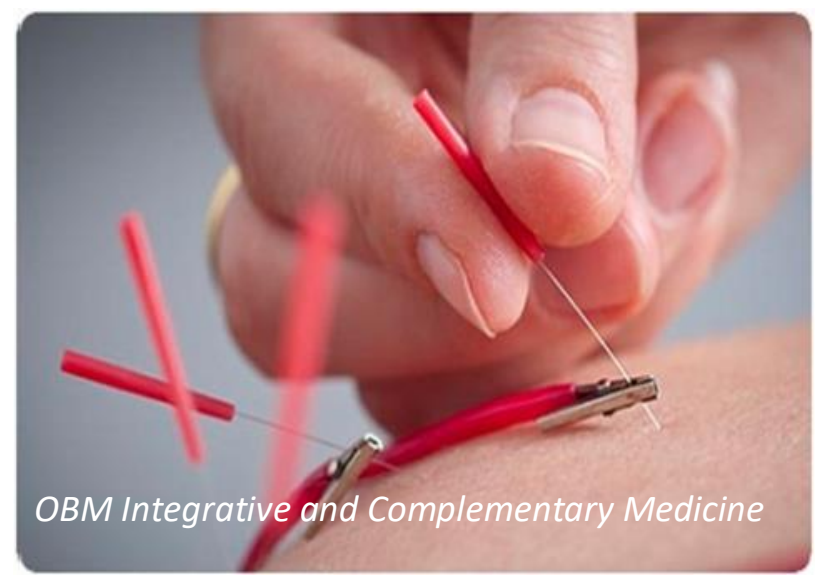

Enjoy OBM Integrative and Complementary Medicine by:

1. Submitting a manuscript

2. Joining in volunteer reviewer bank

3. Joining Editorial Board

4. Guest editing a special issue

For more details, please visit: http://www.lidsen.com/iournals/icm 